\title{
Statyba
}

\section{NEW WAYS OF PACKAGE PROJECTS FOR SMALL AND MEDIUM-SIZED ENTERPRISES (SME) IN GERMANY}

\section{Dr-Ing B. Reichelt}

To cite this article: Dr-Ing B. Reichelt (2001) NEW WAYS OF PACKAGE PROJECTS FOR SMALL AND MEDIUM-SIZED ENTERPRISES (SME) IN GERMANY, Statyba, 7:4, 294-303, DOI: 10.1080/13921525.2001.10531739

To link to this article: https://doi.org/10.1080/13921525.2001.10531739

曲 Published online: 30 Jul 2012.

Submit your article to this journal

III Article views: 52 


\section{NEUE WEGE IM SCHLÜSSELFERTIGBAU FÜR KLEINE UND MITTELSTÄNDISCHE UNTERNEHMEN}

\section{B. Reichelt}

Hochschule für Technik, Wirtschaft und Kultur Leipzig (FH)

\section{Vorwort}

Der in Deutschland anhaltend stagnierende bzw. rezessive Baumarkt stellt kleine und mittelständischen Bauunternehmungen vor große Probleme. Das Überangebot an Bauleistungen führte zu einem Preiskampf und enormen Preisreduzierungen bei gleicher Qualität und gestiegenen Materialpreisen. Weitere Kostenreduzierungen ohne Strukturänderungen sind zwar in begrenztem Maße möglich, diese werden aber zu einer weiteren Erhöhung der Konkurse von Bauunternehmen und zu einer weiteren starken Abnahme der im Bau beschäftigten Arbeitnehmer führen. Diese Situation, die durch die steigende legale und illegale Konkurrenz ausländischer Arbeitnehmer und Bauunternehmen verschärft wird, wird $\mathrm{zu}$ einer Strukturanpassung und $\mathrm{zu}$ neuen innovativen Entwicklungen führen.

Ein der möglichen Lösungswege ist die Ausnutzung von modernen Computer- und Informationstechnologieen in diesem Bereich und das Durchsetzen der neuen Partnerschaftsformen unter den Teilnehmern des Bauprozesses. Die mit der Computerisierung des Bauprozesses verbundenen theoretischen und praktischen Probleme wurden von [1] behandelt. Von einem anderen Autor [2] wird die Computerisierung im einem breiteren Kontext - vom Bau bis zum Betrieb des Bauobjekts behandelt.

Mit den gegenseitigen Beziehungen der Teilnehmer des Bauprozesses befasst sich in seinem Werk auch [3]. In den Abhandlungen der genannten Autoren vermissen wir dennoch die Konkretheit, nicht alle wesentliche Probleme werden besprochen.

Ziel der vorliegenden Behandlung ist die Formulierung von neuen grundlegenden Ideen bei der Ausnuztzung von Computertechnologieen im Bauprozess sowie die Suche nach neuen Partnerschaftsformen.

\section{Schlüsselfertiges Bauen in Deutschland}

Der Schlüsselfertigbau-Markt ist zwar ebenfalls hart umkämpft, die Entwicklungsmöglichkeiten auf diesem wachsenden Marktsegment sind für mittelständische Bauunternehmen jedoch groß.

Obwohl in anderen Ländern ähnliche Konstellationen des Bauens existieren, wird hier kurz auf die deutsche Form, deren Besonderheiten und Probleme eingegangen. Dies soll auch vor dem Hintergrund geschehen, daß zukünftig Firmen aus den EU-Beitrittsländern auf dem deutschen Markt selbständig tätig sein werden.

Beim Schlüsselfertigen Bauen - der Begriff stammt aus dem Hochbau, ist aber auch bei Straßen-, Brückenund Tiefbauten gebräuchlich - wird das gesamte Bauwerk von der Gründung bis schlüsselfertigen Übergabe:

- Komplett und funktionstüchtig;

- von einem Vertragspartner;

- zu einem vertraglich vereinbartem Festpreis und;

- meist bei festgelegtem Fertigstellungstermin erstellt.

Es existieren zwar keine repräsentativen Statistiken, aber die von Kapellmann genannte Größe des Anteils des Schlüsselfertigen Bauens in Deutschland von mindestens 30\% des Bauvolumens erscheint dem Autor glaubhaft [4]. Die Tendenz ist steigend. Hauptsächlich handelt es sich um private Bauaufträge, bei öffentlichen Vorhaben ist diese Form wegen der Anwendung bestimmter Vorschriften des Vergaberechtes eher selten.

Zielgruppe des Schlüsselfertigen Bauens sind Auftraggeber, die eine komplette Bauleistung kaufen, nicht alle Risiken des Bauens tragen und sich nicht mit der Koordinierung von Planern, Bauleitern und Bauunternehmen belasten wollen. Das kann den Bau eines Einfamilienhauses betreffen oder integrierte Vorhaben mit 
Projektentwicklung, Finanzierung, Planung, Ausführung und Betrieb der Immobilie (z. B. BOT-Projekte). Der Beitrag beschränkt sich im weiteren auf für kleine und mittlere Bauunternehmen relevante Fragen.

Vorteil für den Bauherrn gegenüber der sonst üblichen Einzelvergabe in Fachlosen ist neben der Begrenzung des Kosten- und Terminrisikos, ein geringerer Arbeitsaufwand und der Umstand daß während des Bauens und danach nur ein Ansprechpartner haftet.

Der Bauunternehmer kann i.d.R. nicht alle Leistungen des Schlüsselfertigprojektes selbst ausführen und bindet Nach- oder Subunternehmer (NAN) vertraglich, für die er dem Bauherrn gegenüber haftet. Er wird Generalunternehmer (GU) genannt, wenn er einen Teil der Bauleistung selbst ausführt und Generalübernehmer (GÜ), wenn er alle Leistungen an Nachunternehmer vergibt. Wird die gesamte Planung durch das Bauunternehmen übernommen spricht man von einem Totalunter- nehmer. Abbildung 1 stellt die Vertragsbeziehungen des Generalunternehmers und Totalunternehmers im Gegensatz zur in Deutschland typischen Einzelvergabe dar.

Die entsprechenden Verträge des Schlüsselfertigbaus sind Pauschalverträge meist auf der Basis funktionaler Ausschreibungen. Je nach Vorgabe des Bauherrn kann die komplette Bauleistung um Teile der in Deutschland traditionellen Architekten- und Ingenieurplanung erweitert werden. Diese kann der Generalunternehmer dann selbst ausführen oder an Planungsbüros in seinem Auftrag weitergeben. Da das Vorhaben funktionstüchtig übergeben werden muß, übernimmt er somit auch Teile des Planungsrisikos. Die mittlerweile übliche Schnittstelle im Schlüsselfertigbau ist die Übergabe der Genehmigungsplanung, die durch den Architekten des Bauherrn erstellt wurde. Der Generalunternehmer übernimmt dann Ausführungsplanung und Teile der Tragwerks- und Haustechnikplanung.
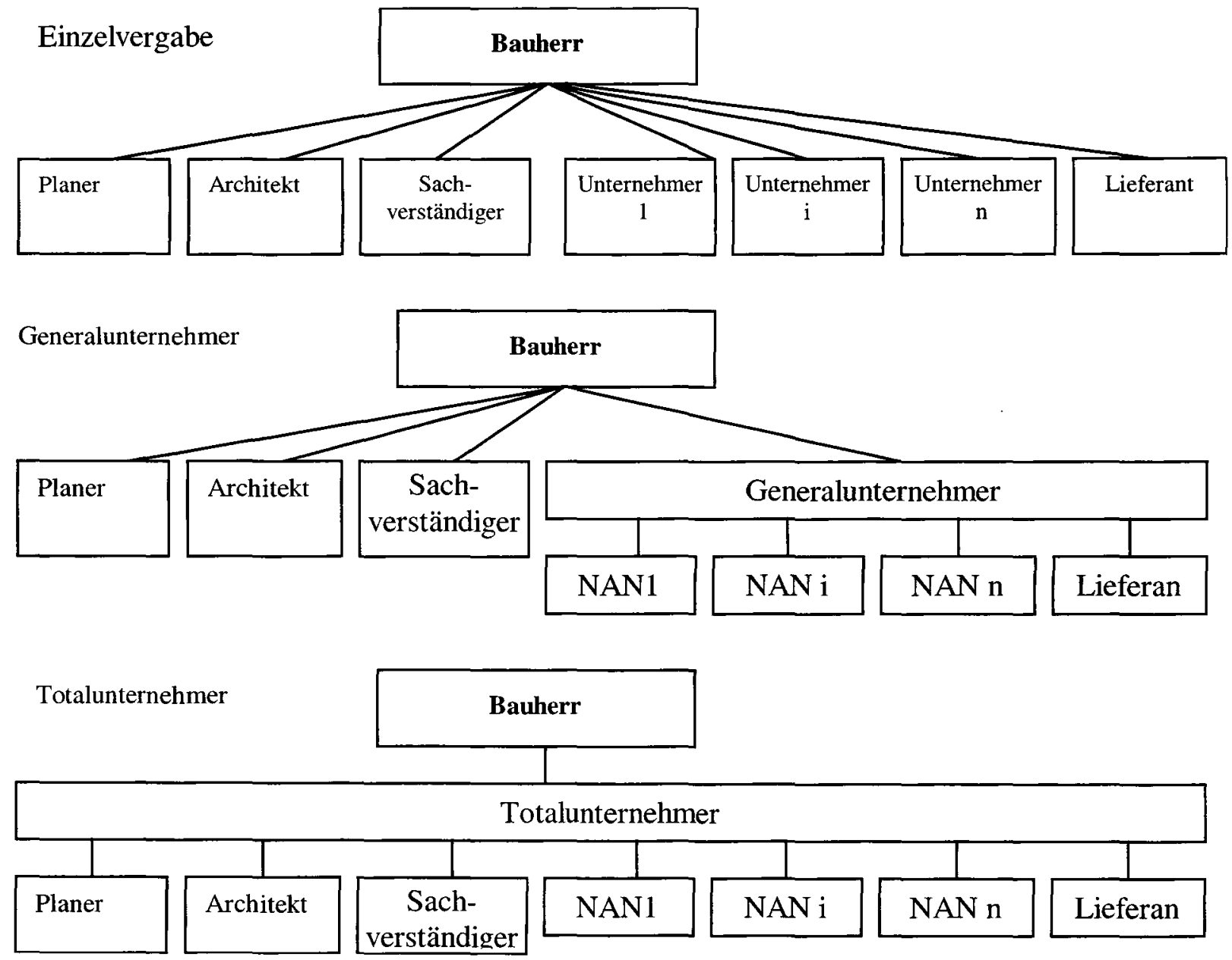

Abb. 1. Vertragsbeziehungen Einzelvergabe und Formen des schlüsselfertiges Bauen

Fig 1. Relation between individual allocation of orders and forms of package projects 
Abbildung 2 versucht die Bandbreite der Leistungen eines Bauunternehmers, der als GU auftritt, darzustellen.

Die Leistung und deren Qualität wird i. d. R. durch Entwurfszeichnungen und eine mehr oder weniger detaillierte Baubeschreibungen spezifiziert. Innerhalb dieser Vorgaben, unter Berücksichtigung der sog. Allgemein Anerkannten Regeln der Baukunst, hat der GU einen relativ breiten Ausführungsspielraum, den er zu seinem Kostenvorteil u. a. zu nutzen versucht durch:

- soweit zulässig kostengünstige alternative Baukonstruktionen und Ausführungsvarianten;

- eine fertigungsgerechte Planung;

- weitgehend parallelen Steuerung seiner Planung/ Arbeitsvorbereitung mit der Ausführung;

Leistungsumfang klassisches Modell

\begin{tabular}{|c|l|l|l|}
\hline & Bauherr & Architekt & $\begin{array}{c}\text { Bauun- } \\
\text { ternehm. }\end{array}$ \\
\hline $\begin{array}{c}\text { Projekt- } \\
\text { entwicklung }\end{array}$ & & & \\
\hline $\begin{array}{c}\text { Bauprogramm/ } \\
\text { Grundlagenermittl. }\end{array}$ & & & \\
\hline Vorentwurf & & & \\
\hline $\begin{array}{c}\text { Entwurf/ } \\
\text { Genehmigungspl. }\end{array}$ & & & \\
\hline $\begin{array}{c}\text { Ausfuihrungspl./ } \\
\text { Vergabe }\end{array}$ & & & \\
\hline $\begin{array}{c}\text { Bauüberwachung } \\
\text { Herstellung des } \\
\text { Bauwerks }\end{array}$ & & & \\
\hline Betrieb & & & \\
\hline
\end{tabular}

- seine Kenntnisse des Baumaterialienmarktes und der Nachunternehmermarktes.

Das kann ein Nachteil für den Bauherrn sein, der nach Vertragsunterzeichnung nur noch geringen Einfluß auf Details hat, die im Vertrag nicht beschrieben wurden und Änderungen des Entwurfs i. d. R. teuer bezahlen muß.

\section{Probleme der Schlüsselfertigen Bauens}

In den letzten Jahrzehnten bildeten sich im Schlüsselfertigbau verschiedene Probleme heraus, die sich durch die eingangs geschilderte negative Wirtschaftslage verschärfen. Im folgenden sollen die $\mathrm{m}$. E. wesentlichen Aspekte besprochen werden.

Typische Leistungsverteilung Generalunternehmer

\begin{tabular}{|c|l|l|l|}
\hline & Bauherr & Architekt & $\begin{array}{c}\text { Bauun- } \\
\text { ternehm. }\end{array}$ \\
\hline $\begin{array}{c}\text { Projekt- } \\
\text { entwicklung }\end{array}$ & & & \\
\hline $\begin{array}{c}\text { Bauprogramm/ } \\
\text { Grundlagenermittl. }\end{array}$ & & & \\
\hline Vorentwurf & & & \\
\hline $\begin{array}{c}\text { Entwurf/ } \\
\text { Genehmigungspl. }\end{array}$ & & & \\
\hline $\begin{array}{c}\text { Ausfuihrungspl./ } \\
\text { Vergabe }\end{array}$ & & & \\
\hline $\begin{array}{c}\text { Bauüberwachung } \\
\text { Herstellung des } \\
\text { Bauwerks }\end{array}$ & & & \\
\hline Betrieb & & & \\
\hline
\end{tabular}

BOT-Modell

\begin{tabular}{|c|l|l|l|}
\hline & Bauherr & Architekt & $\begin{array}{c}\text { Bauun- } \\
\text { ternehm. }\end{array}$ \\
\hline $\begin{array}{c}\text { Projekt- } \\
\text { entwicklung }\end{array}$ & & & \\
\hline $\begin{array}{c}\text { Bauprogramm/ } \\
\text { Grundlagenermittl. }\end{array}$ & & & \\
\hline $\begin{array}{c}\text { Vorentwurf } \\
\text { Genehmigungspl. }\end{array}$ & & & \\
\hline $\begin{array}{c}\text { Ausführungspl./ } \\
\text { Vergabe }\end{array}$ & & & \\
\hline $\begin{array}{c}\text { Bauüberwachung } \\
\text { Eerstellung des } \\
\text { Bauwerks }\end{array}$ & & & \\
\hline Betrieb & & & \\
\hline
\end{tabular}

Abb. 2. Darstellung des möglichen Leistungsumfangs des Bauunternehmers im Schlüsselfertigbau

Fig 2. Possible services that could be offered by building enterprises in the framework of a packaging project 
Mangelhafte Planung des AG. Die Planungsunterlagen, die der Auftraggeber dem GU übergibt, sind häufig mangelhaft, in sich oder zu Behördenauflagen widersprüchlich oder nicht ausführbar. Die Ursachen sind vielfältig:

- der Architekt fühlt sich für alles was nach der Genehmigung kommt nicht verantwortlich, insbesondere, wenn sein Honorar nicht angemessen ist,

- die Ausbildung der Planer ist stark von der Ausführung entfernt,

- wegen mangelnder Erfahrung überblickt der Bauherr die Folgen nicht.

Den Anpassungsaufwand der Planung bekommt der GU in der Regel nicht vergütet, das Kalkulationsrisiko ist entsprechend groß. Diese schlechte Vorbereitung seitens des Auftraggebers schließt nicht selten die Finanzierung ein, was u. a. Zahlungsausfälle zur Folge hat.

Schlechte Bauvorbereitung des GU. Meist muß in sehr kurzer Zeit für eine komplexe Bauaufgabe ein Angebot erstellt werden, das konkurrenzfähig ist und die Weichen für Erfolg oder Verlust stellt und das unter dem Vorzeichen der o.g. Planungsvorgaben. Viele GU besonders im mittelständischen Bereich kalkulieren deshalb trotz der Risiken und der terminlichen, wirtschaftlichen und technischen Verantwortung ihre Vorhaben in der Angebotsphase nicht umfassend genug. Diese Arbeitsweise setzt sich durch eine traditionellen Nachlässigkeit fort, was dazu führt, daß z. B. in der Sanierung während des Bauens sehr viel operativ geregelt wird. Die Folge ist Zeitdruck und schlechte Qualität.

Wirkung auf die Nachunternehmen. Der GU versucht üblicherweise viele dieser Probleme und Risiken auf die Nachunternehmer zu übertragen. Oft entscheidet bei der Vergabe allein der Preis, was auch hier zu mangelhafter Qualität, längeren Projektdurchlaufzeiten, den Einsatz von Schwarzarbeit und nicht selten zu Konkursen führt. Der Nachunternehmer muß sich als Kostenführer etablieren und hat kaum die Möglichkeit sich technisch-konstruktiv oder fertigungstechnisch $\mathrm{zu}$ spezialisieren und somit seine Zukunft zu sichern. Er verliert mehr und mehr seine Marktanpassungsfähigkeit.

Die Zunahme des Schlüsselfertigbau ist für kleine Unternehmen, die in Deutschland den wesentlichen Teil des Bauvolumens bringen, überhaupt problematisch.
Ihnen wird die unmittelbare Teilnahme am Wettbewerb um Aufträge erschwert.

Reibungsverluste. In der Planungsphase und stärker auf der Baustelle ist unter diesen Vorzeichen eine offene Zusammenarbeit zwischen den Vertragspartnern kaum möglich. Abstimmungsprobleme zwischen Bauherren, Architekten und den Bauunternehmen, die unterschiedliche Interessen verfolgen, führen zu Reibungsverlusten. Unterschwellige Konflikte und offene Feindseligkeit sind an der Tagesordnung und führen $\mathrm{zu}$ kostenträchtigen Störungen.

Wegen der niedrigen Baupreise pflegt das Bauunternehmen das Nachtragsmanagement, was dazu führt, daß der Bauherr Claim-Management betreibt. Mißtrauen herrscht vor und Zusammenarbeit wird formal durch Vertragsbedingungen geregelt. Man beschäftigt sich auch bei kleinen Vorhaben immer mehr mit baurechtlichen Problemen, sichert sich gegenseitig baurechtlich ab, um Haftungsansprüchen, Vertragsstrafen usw. zu entgehen. In der Regel investiert jeder Beteiligte mehr Energie in bauvertragsrechtliche Fragen als in die Verbesserungen des Bauens - man kann hier von einer „Verrechtlichung des Bauens" sprechen.

Wenn auch nicht alle Baustellen durch diese Verhältnisse geprägt sind, so belasten sie auch in abgeschwächter Form das Beziehungsgeflecht der Beteiligten außerordentlich.

\section{Generelle Lösungsansätze für kleine und mittel- ständische Bauunternehmen}

Der gegenwärtig in Deutschland eingeschlagene Weg des Schlüsselfertigen Bauens führt offensichtlich weder zu einer Verbesserung der Marktchancen der kleinen und mittelständischen Bauunternehmen noch zu einer signifikanten Kostenreduzierung des Bauens. Nach Meinung des Autors sind vier prinzipielle Auswege denkbar:

- Kundenorientierte Angebote und Auftragsabwicklung;

- Rationellere Arbeitsorganisation beim GU, angelehnt an das prozeßorientierte Qualitätsmanagement;

- Einsatz durchgängiger EDV-Lösungen;

- Verbesserung der Kooperation zwischen den am Bau Beteiligten. 
Die Erfahrung der letzten Jahre in der Bauwirtschaft ist, daß qualitativ hochwertig erstellte Bauwerke kein Garant mehr für den langfristigen Erfolg eines Unternehmens sind - sie müssen auch preisgünstig, schneller erstellt und den veränderten Kundenanforderungen angepaßt werden.

Es müssen Leistungsangebote entwickelt werden, die vom Umfang und vom Risiko her den Wünschen und Vorstellungen des Bauherrn entsprechen - dies kann von der Projektentwicklung bis zum Gebäudemanagement reichen. Es kommt auf das Anbieten des richtigen Produktes und die Zufriedenheit des Bauherrn an. Das schließt individuelle Beratung und Information vor und während der Vertragsabwicklung ein, z. B. zu neuen kostengünstigeren Alternativen von Materialien oder Ideen zur besseren Vermietbarkeit. Dazu bedarf es Vertrauen, das sich durch fachliche Kompetenz, Zuverlässigkeit, Vertragstreue und dem gemeinsamen Willen eine erfolgreiche Baumaßnahme zu realisieren auszeichnet.

Rationellere Arbeitsorganisation beim GU bedeutet Neugestaltung der gesamten betrieblichen Prozesse und damit verbunden die Verbesserung der internen Kommunikation.

Besonders Mitarbeiter der unteren Führungsebene einschließlich der Facharbeiter müssen mehr Verantwortung übernehmen und selbständiger arbeiten. Diese Maßnahmen führen häufig zum Hinterfragen und zur Änderung des Führungsstils im Unternehmen.

Beim Einsatz durchgängiger EDV-Lösungen geht es um eine effektive Unterstützung des GU und der NAN über den gesamten Planungs- und Bauproze $\beta$, um Aktualität und Schnelligkeit in bezug auf Information und Kommunikation in Planung, Realisierung und Gewährleistung.

Der Weg des nahtlosen Ineinandergreifens der Daten aller Planer und Ausführenden (CAD, AVA, Kalkulation, Abrechnung) kann dabei wegen der üblichen funktionalen Ausschreibung und der fehlenden hardware- und softwareseitigen Voraussetzungen beim Nachunternehmer nicht vordergründig betrachtet werden. Schnittstellen zur Übernahme von Daten vor- und nachgelagerter Prozesse, zur Integration der Planungsleistungen von Nachunternehmern insbes. des Haustechnikbereiches, zur Übergabe der Daten für die Zwecke des Bauherrn, z.B. für das Facility Management, sind jedoch notwendig.

Außer bei Arbeitsgemeinschaften hat eine partnerschaftliche Kooperation zwischen verschiedenen selbstän- digen Unternehmen in Deutschland keine Tradition. Generell sind 4 verschiedene Richtungen zu erkennen:

- Arbeitsgemeinschaft (ARGE);

- Dach-ARGE oder Konsortium;

- Partnering bzw. Garantierter Maximalpreises (GMP);

- partnerschaftliche Kooperation zwischen den am Bau Beteiligten bei Beibehaltung der prinzipiellen vertraglichen Beziehungen.

Die ARGE-Modelle und das GMP-Modell werden als bekannt vorausgesetzt. Die partnerschaftliche Kooperation versucht die am Bau Beteiligten, am Erfolg $\mathrm{zu}$ interessieren. Dieser Anreiz kann liegen in:

- der Aufteilung eines zusätzlichen Gewinnes durch gemeinsam erarbeitete kostengünstigere Verfahren,

- einer wegen der neuartigen Kooperation sichereren erfolgreichen Abwicklung des Bauvorhabens u.a. wegen verminderter Abstimmungsprobleme hinsichtlich Ablauf und Koordination,

- einer verbesserten Akquisition,

- der partizipativen Einflußnahme auf den Bauprozeß zur besseren Abstimmung der eigenen Belange mit denen des Projektes, um dadurch eine höhere technologische Sicherheit zu erreichen.

\section{Lösungsansatz Partnerschaftliche Kooperation}

Anfang 2001 wurde durch die HTWK Leipzig, Fachbereich Bauwesen, Lehrbereich Baubetrieb und einem mittelständischen Bauunternehmen, das ca. 100 Mitarbeiter beschäftigt, ein Forschungsprojekt begonnen, das im Kern den Ansatz der Verbesserung der Kooperation zwischen GU und NAN in Verbindung mit einer breiten Unterstützung durch Informations- u. Kommunikationstechnologie hat [5]. Es sollen durch kreative Zusammenarbeit kostengünstigere und qualitativ hochwertigere Lösungen gefunden, die Zufriedenheit der Beteiligten erhöht und damit die Marktchancen für kleine und mittelständische Unternehmen verbessert werden. Dieses Modell ist prinzipiell auch für die Zusammenarbeit mit Bauherren, Planern und Lieferanten einsetzbar.

Im weiteren soll auf folgende Aspekte des Modells eingegangen werden:

- Kooperationskonzept;

- EDV-Konzept;

- Projektablauf; 
- Erfolgsfaktoren der Einführung.

A Kooperationskonzept. Die Kooperation ähnelt vom Grundverständnis her einer Symbiose unterschiedlich großer Lebewesen (hier Unternehmen), die voneinander profitieren und nicht oder nur schlecht ohne einander leben können.

Das notwendige partnerschaftliche Verhalten muß von allen gelernt und (am Anfang vom Genralunternehmer vor-) gelebt werden. Es gilt, ein geeignetes Arbeitsumfeld zu schaffen, das die kreative Suche nach innovativen kosten- und zeitersparenden Lösungen fördert. Voraussetzung ist eine offene, transparente, vertrauensvolle und motivierte Zusammenarbeit gemäß einer Gewinner- Gewinner-Strategie. Dabei wird eine Art Netzwerk entstehen, das auch außerhalb aktiver Verträge wirksam ist

Der NAN muß eine Gesamtprojektsicht entwickeln und in das Risikomanagement eingebunden werden. Das heißt unter anderem, daß ihm mehr Selbständigkeit und Verantwortung übertragen und die Kontrolle schrittweise verringert wird. Die intensive gemeinsame Entscheidungsvorbereitung des GU mit den NAN muß bereits während der Angebotskalkulation erfolgen.

Alle Beteiligten tauschen Informationen über ein computergestütztes Informations- und Kommunikations- system aus und legen so die notwendigen Daten offen. Inwieweit das auch sensible Daten betrifft, hängt von der Art der entstehenden Zusammenarbeit und der Gewinnaufteilung $a b$, die transparent erfolgen muß. Abbildung 3 zeigt das Prinzip der Kommunikationsstruktur.

Das „Generalunternehmerprinzip“ wird weiterentwickelt. Der Generalunternehmer wird mehr Verantwortung gegenüber seinen Nachunternehmern übernehmen. Das bedeutet, $\mathrm{da} B$ er sich auch für die internen Prozesse des Nachunternehmers interessiert und Hilfen anbietet, die sich beziehen können auf:

- die strategische Unternehmensentwicklung des Nachunternehmers z. B. Unterstützung der Einführung des Qualitätsmanagements;

- das Finden und Einführen innovativer Methoden des Bauens für die Projekte des GU, die gleichzeitig eine Verbesserung der Marktchancen des NAN bedeuten;

- Beteiligung des GU an Entwicklungen, die der Nachunternehmer nicht allein finanzieren kann.

Erfolgreiche Kommunikation und Zusammenarbeit werden wesentlich von der Projektkultur geprägt. Diese umfaßt die Grundannahmen, Wertvorstellungen, Einstellungen, Verhaltensregeln, Normen usw. der am

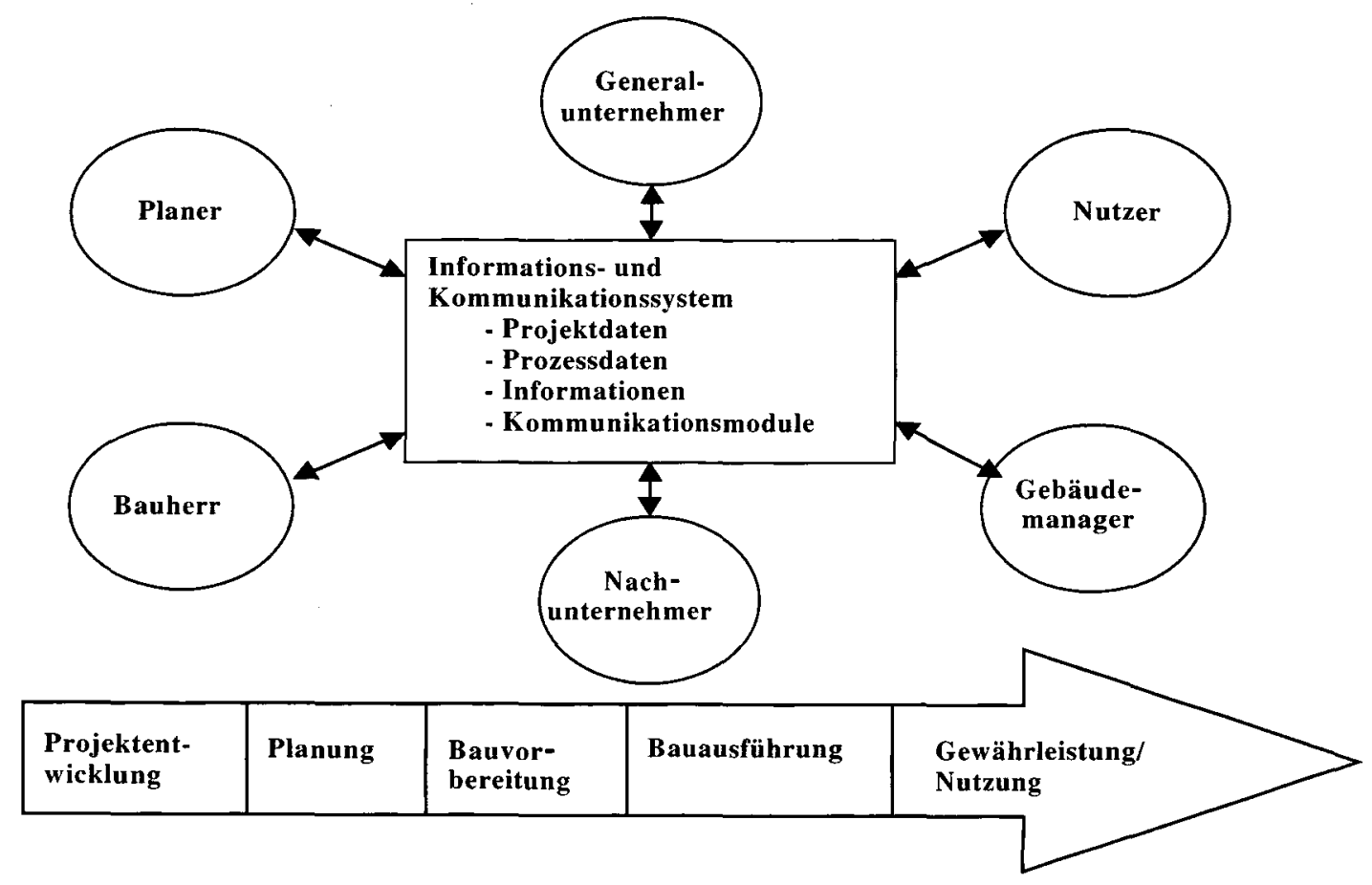

Abb. 3. Kommunikationsstruktur bei der Partnerschaftlichen Kooperation

Fig 3. Communication structure within partnering 
Projekt beteiligten Personen und wird durch Führungsstil, Organisations- und Managementmethoden getragen. Projektkultur hat erheblichen Einfluß auf Entstehung und Behebung von Konflikten innerhalb von Projekten. Die Herausbildung einer entsprechenden die Zusammenarbeit und Innovation fördernden Projektkultur im Netzwerkes ist fester Bestandteil des Modells.

Auch Verträge müssen entsprechend ausgestaltet werden, da sie darauf ausgerichtet sind, unter der jeweiligen Projektkultur erfolgreich zu sein. Vertrag und Projektkultur bedingen einander und beeinflussen sich gegenseitig.

Wie kann die Projektkultur konkret entwickelt und gestaltet werden? Hier nur einige Beispiele:

- Entwickeln und Einführen von Organisations- und Führungsmethoden, die partnerschaftliches Miteinander fördern z. B. Moderation, spezielle Kurzberatungen, Leistungspakete, die auf selbständige Erfüllung ausgerichtet sind;

- Offenlegen der unternehmerischen und persönlichen Interessen der Beteiligten;

- Ständiges gemeinsames Suchen nach neuen Lösungen, generelles Offenhalten gegenüber neuem, einschl. Integration von Unternehmen.

Die Projektkultur wird sich mit der Zeit herausbilden und auf das Verhalten zurückwirken, daher:

- ist eine ständige bewußte Pflege und Weiterentwicklung durch die Beteiligten notwendig z. B. durch entsprechende Feed-Back-Möglichkeiten;
- müssen Vorkehrungen getroffen werden, daß Probleme/Störungen so früh wie möglich erkannt und beseitigt werden können.

Eine wichtige Person bzw. Stelle, die den Prozeß der Bildung der Projektkultur und der Abwicklung der Bauvorhaben beobachtet, aktiv begleitet und für einen konfliktarmen Bauablauf sorgt, ist die eines Mediators oder Coaches. Dieser entwickelt das System der Kooperation weiter, erkennt und löst Konflikte innerhalb der Kooperation. Bei jedem Bauvorhaben sorgt er für die Abstimmung des Informationsflusses, des Berichtswesens und der Kompetenzen, moderiert Beratungen, die für die Zusammenarbeit wichtig sind, und richtet das Informationssystem ein und pflegt es.

Aus den Aufgaben ist erkennbar, daß der Mediator weitgehend neutral, von allen als Vertrauensperson (Ansprechpartner) anerkannt und fachlich kompetent sein muß. Abbildung 4 zeigt den strukturellen Zusammenhang der Beteiligten in einem Organigramm.

$B$ EDV-Konzeption für die Partnerschaftliche Kooperation. Ziel ist die Unterstützung der beschriebenen Kooperationsform von der Arbeitsvorbereitung / Planung bis zur Baustellenabrechnung / Gewährleistung d. h. ein leistungsfähiges Kommunikationsnetz, das das Bauwerk und den Prozeß des Planens und Bauen abbildet und eine gezielte, effektive Informationssteuerung gewährleistet. Es entsteht eine Art zentrales, virtuelles Projekt- und Organisationshandbuch, das von allen Beteiligten entsprechend dem Baufortschritt, den

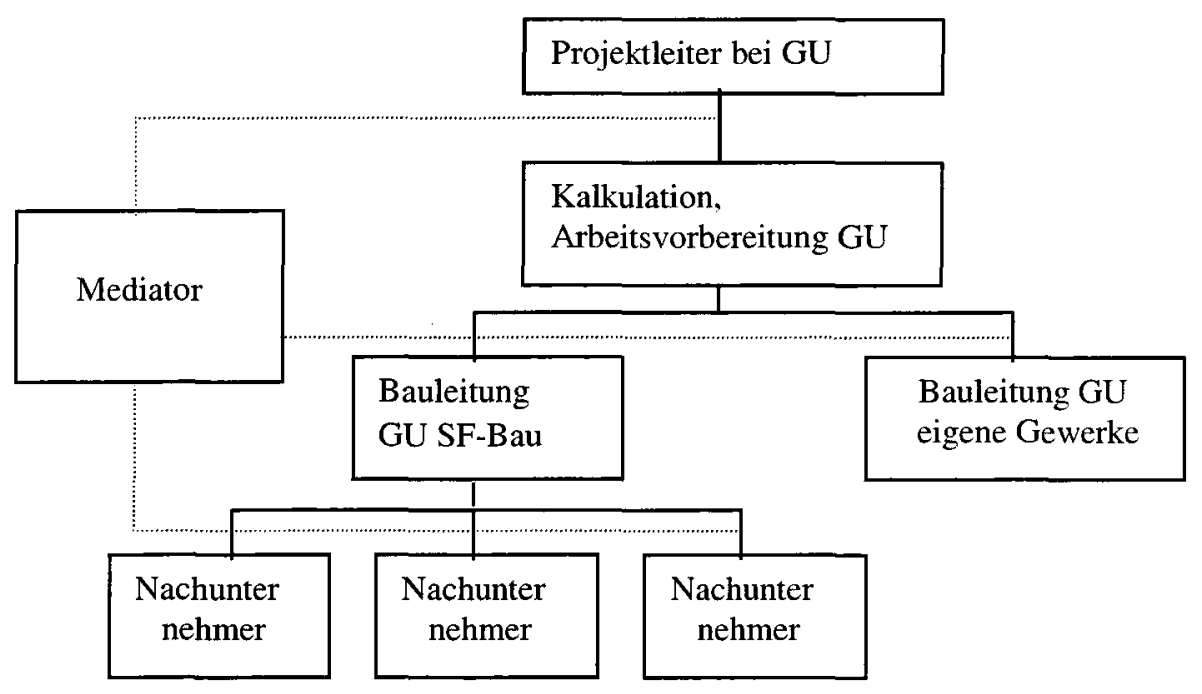

Abb. 4. Organigramm für die Baustellensteuerung und-vorbereitung

Fig 4. Outline showing preparation and control of building sites 
Aufgaben und Kompetenzen weiterentwickelt wird.

Das Bauwerksmodell hat eine Vielzahl von erklärenden technischen, wirtschaftlichen, organisatorischen und rechtlichen Daten und muß somit alle Informationen vorgelagerter Prozesse übernehmen können. Es muß mit den Leistungsdaten in geeigneter Weise verknüpft werden, in der Gliederungstiefe flexibel sein und Änderungen jederzeit einfach zulassen. Erste Untersuchungen zum Datenmodell liegen vor [6].

Das Prozeßmodell bildet den Kommunikations- und den Bauprozeß ab, d. h. Plan-, Ist- und Analysedaten, Ablauf der Projektorganisation, Berichtswesen usw. Die Daten werden durch feste Verantwortlichkeiten der einzelnen Beteiligten aktuell gehalten.

Die EDV-Plattform muß gestatten, die Nachunternehmer bei der Datenerfassung und -nutzung direkt einzubeziehen. Der Informationsverbund ist über mehrere Standorte (Baustellen, NAN-Büros) hinweg notwendig und erfordert Informations- und Kommunikationstechnik, wie etwa Internettechnologie und Methoden des e-Commerce, die ein redundanzfreies Arbeiten ermöglichen [7].

$C$ Der Proze $\beta$. Der Prozeß bündelt alle beschriebenen Aspekte in einem Ablauf, der einerseits für alle Vorhaben gleich und andererseits flexibel auf jedes Bauvorhaben zugeschnitten sein muß. Dazu werden Instrumente und Methoden des Qualitätsmanagements genutzt. Mit diesem Ablauf sollen Störungen und Mängel minimiert werden $z$. B. indem eine wirtschaftliche und möglichst exakte Planung gesichert wird und die Kenntnis von Risiken und Störungsmöglichkeiten ein vorausschauendes Handeln aller Beteiligten erreicht. Der Prozeßablauf versucht die Nachunternehmer und die Mitarbeiter regelmäßig in die Entscheidungen mit einzubeziehen, um zur gleichen Projektvorstellung und $\mathrm{zu}$ klaren Aufgabendefinitionen und Kompetenzen zu kommen.

Der prinzipielle Projektablauf wird in Abb. 5 in den wichtigsten Teilen skizziert.

Zu diesem Prozeß gibt es Standardprozeduren wie:

- Änderungen durch Störungen, Bemusterungen oder Bauherrenwünsche;

- Angebote von Sondervorschlägen und Verbesserungen;

- Soll-Ist-Vergleiche und die notwendigen Reaktionen;

- Verhalten bei Mängeln, Fehlern usw.

\begin{tabular}{|c|}
\hline ANGEBOT \\
\hline Marktkontakt, Akquisition \\
\hline Ausschreibungseingang \\
\hline Erfassen der Grundkennwerte \\
\hline Kalkulationsvorentscheid \\
\hline Kalkulation Rahmenbedingungen \\
\hline Abfrage und Beratung mit NAN \\
\hline Angebotskalkulation \\
\hline Abgabe Angebot \\
\hline Vertragsverhandlung und - abschlu $\beta$ \\
\hline BAUVORBEREITUNG \\
\hline Interner Projektstart \\
\hline Projektstart mit Nachunternehmern \\
\hline Auftragskalkulation GU(parallel bei Nachunternehmer) \\
\hline Termin-, Kosten-, Qualitätsplanung \\
\hline Projektplanung und Optimierung der Planung \\
\hline $\begin{array}{l}\text { Arbeitsvorbereitung unterBerücksichtigung der NAN- } \\
\text { Vorstellungen }\end{array}$ \\
\hline BAUREALISIERUNG \\
\hline Baustart intern \\
\hline Baustart extern - Einweisen der AN des NAN \\
\hline Baudurchführung \\
\hline Bauablaufkontrolle/-steuerung, Abrechnung \\
\hline Abnahme und Dokumentation \\
\hline NACHBEREITUNG/GEWÄHIRLEISTUNG \\
\hline Nachkalkulation/ Auswertung \\
\hline Projektbeurteilung durch Beteiligte \\
\hline Gewährleistung \\
\hline Kundenbetreuung \\
\hline
\end{tabular}

Abb. 5. Projektablauf aus Sicht des GU bei Einbeziehung der Nachunternehmer

Fig 5. Stages of the project from the point of view of the main contractor including sub-contractors

Problem ist die effektive Bewältigung der Vielzahl von Abstimmungen. Hier müssen neue Beratungsformen getestet werden, z. B. Videoconferencing, geeignete kurze Beratungstechniken u.a. Diese Details sind während des Projektes zu entwickeln, zu festigen und einem Methodenpool zuzuführen. 


\section{Erfolgsfaktoren der Einführung der Partner-} schaftlichen Kooperation. Die Verwirklichung des Vorhabens wird auf verschiedene Schwierigkeiten treffen.

Einer zu geringen Akzeptanz unter den Beteiligten wird begegnet, indem bisher zwei NAN, die ähnliche Vorstellungen von Zusammenarbeit haben, am Projekt mitarbeiten. Außerdem werden die Interessen der am Bau Beteiligten innerhalb eines Teilprojektes detailliert untersucht. Um dem Anwenderkreis der kleinen und mittleren Unternehmen gerecht zu werden, soll das EDV-System zwar durchgängig aber ergonomisch einfach gestaltet sein.

Etwa ein Jahr nach Projektbeginn ist ein Feldversuch vorgesehen, der sich auf ein oder zwei Vorhaben mit eingegrenztem Leistungsumfang bezüglich Gewerken und beteiligten Firmen beschränkt, dabei sollen sowohl das Kooperationskonzept als auch das EDV-Konzept Berücksichtigung finden, die Erfahrung des Feldversuches fließen in den Prototyp ein. Für die endgültige Ausprägung des Systems wird ein spezielles Schulungskonzept geschaffen, das vorrangig auf Lernen von Verhaltensweisen durch Plan- und Rollenspiel abzielt.

\section{Ausblick - Die zukünftige Verwertung}

Der Autor geht davon aus, daß die Partnerschaftliche Kooperation in Zukunft eine wichtige Rolle im Schlüsselfertigbau spielen wird. Das konzipierte Modell kann nach der Weiterentwicklung des Prototypes und der Optimierung des Informationssystems eine Marktchance im Bereich kleine und mittelständische Unternehmen haben. Das Methodenwissen und die Software fließen in ein Produkt ein, das als Franchise-Lösung verwertet werden soll. Eingeschlossen sind eine Begleitung des interessierten Generalunternehmers, seiner Nachunternehmer und ggf. von Bauherrn einschließlich Schulungsmaßnahmen, programmtechnische Unterstützung, Testläufen, Konflikt- und Störungsbeseitigung usw. In den ersten Projekten ist der Einsatz des Mediators durch den Franchisegeber erforderlich.

Eine wissenschaftliche Weiterentwicklung der Methode und der Software kann ein Institut für „Partnerschaftliche Kooperation im Bauwesen“ leisten, das sich aus dem Verkauf der Franchise-Lösungen finanziert und zusätzlich außergewöhnliche Probleme behandelt, Nachunternehmer und Generalunternehmer beim Finden neuer Ideen unterstützt und durch Öffentlichkeitsarbeit eine Breitenwirkung für die Methode erreicht.

\section{Literatur}

1. J. Nävy. Facility-Management: Grundlagen, Computerunterstützung, Einführungsstrategie, Praxisbeispiel. Springer, 1998. $296 \mathrm{~S}$.

2. H. Braun, P. Haller, E. Oesterle. Erfolg in der Immobilienbewirtschaftung. Springer, 1996. $162 \mathrm{~S}$.

3. H. Kahlen. Management des ganzheitlichen Bauens. Düsseldorf: Werner, 1999. $332 \mathrm{~S}$.

4. K. D. Kapellmann. Schlüsselfertiges Bauen: Rechtsbeziehungen zwischen Auftraggeber, Generalunternehmer, Nachunternehmer, Werner-Verlag Düsseldorf 1997. S. 2.

5. HTWK Leipzig und Euro-Consult GmbH Leipzig Partnerschaftliche Kooperation mit mittelständischen Generalunternehmen bei Instandsetzung und Modernisierung im Wohnungsbau Projektskizze zum Verbundprojekt des BMBF Programms „Bauen und Wohnen“, Förderschwerpunkt „Bauforschung und Technik, Mai 2001.

6. M. Vogt. Entwicklung eines Datenmodells für die Abwicklung von GU-Prozessen im Bereich Altbausanierung; Diplomarbeit HTWK Leipzig 2000.

7. F. Exter. Lösungsansätze für ein Informations- und Kommunikationssystem auf Basis von ERP- u. e-Business- Konzepten; unveröffentlichtes Manuskript, Leipzig 2001 .

Iteikta $200105 \quad 10$

\section{NAUJOS SMULKIUUJU IR VIDUTINIU STATYBOS IMONIU PERSPEKTYVOS}

\section{B. Reichelt}

\section{Santrauka}

Šiuo metu Vokietijos statybos pramoné išgyvena nuosmukị. Kad išsilaikytu. smulkiosios ir vidutinès imonès turi prisitaikyti prie besikeičiančios situacijos. Vienas iš problemu sprendimu būdų - tapti pagrindiniu rangovu. Iki šiol jais būdavo didžiosios statybos imonès.

Aprašomos problemos, kurios kyla, kai smulkiosios ir vidutinès imonés imasi projektu paketo. Suformuluota principiné idèja, kurios esmé - intensyvus kompiuteriu naudojimas visuose statybos procesuose ir nauju partnerystés formı, kurios dar mažai žinomos Vokietijoje, taikymas.

Autorius isitikinęs, kad ateityje bus labai svarbu partneriu kooperavimasis, statant visiškai užbaigtus ir priduoti parengtus objektus. Modelis, kurio koncepcija siūloma, patobulinus prototipa ir optimizavus informacinę sistema, yra perspektyvus smulkiujų ir vidutinių imonių atžvilgiu. Metodinèms žinioms ir programinei ịrangai susiliejus $\mathfrak{i}$ vieną produktą gaunamas naujas sprendimas - franšizè - integruota darbo su suinteresuotu generaliniu rangovu, jo subrangovais ir, reikalui esant, 
statybos šeimininku, sistema iskaitant apmokymus, parama programinès technikos priemonèmis, kompiuterini testavima. konfliktų ir sutrikimu šalinimą ir pan. Pirmuosiuose projektuose franšizès davejas privalo skirti tarpininką.

Metodikos ir programines irangos mokslinio tobulinimo darbus gali atlikti Statybos partneriy kooperavimosi institutas, kuris užsidirba lèšu iš franšiziụ pardavimo ir papildomai užsiima netipinių problemų sprendimu, igyvendina subrangovų bei rangovu iškeltas naujas idejas, populiarina metodiką visuomenèje.

\section{NEW WAYS OF PACKAGE PROJECTS FOR SMALL AND MEDIUM-SIZED ENTERPRISES (SME) IN GER- MANY}

\section{B. Reichelt}

\section{Summary}

In Germany the building industry is in recession at the moment. Especially the SMEs have to adapt to different situation in order to survive. One possible strategy could be taken on orders as main contractors. This has until now been donne dominantely by large building enterprises.

The author describes problems and chances which arise if the SMEs take on package projects. He develops a strategic idea that is based on intensive use of computers in all processes and on new forms of cooperation (partnering) that is less known in Germany.

The author is convinced that in future the collaboration of partners will play a significant role in the construction of structures that are completely finished and ready for sale. After the improvement of the prototype and optimisation of the information system, the proposed model has a good potential in the field of small and medium business units. After the methodological knowledge and software blend into one product, a new solution will be received, viz the franchise. The integrated system of working with an interested contractor general, his subcontractors and, if necessary, the construction master, that includes training, software support, software test runs, elimination of conflicts and faults, etc. In the first projects the franchiser must provide the intermediator.

The Institute of Collaboration of Construction Partners that earns its income from the sale of franchises may perform scientific improvement of methods and software; the Institute is also engaged in dealing with atypical problems, support of subcontractors and contractors in their search for new ideas, as well as in the promotion of its methods using public relations.

Bernd REICHELT. Dr-Ing, Professor. Dept of Civil Engineering and Architecture. Leipzig University of Applied Science (HTWK), Karl-Liebknecht-Str. 132, D-04277 Leipzig, Germany. E-mail: reichelt@fbb.htwk-leipzig.de

Dr-Ing (1989); 1984-92 University of Technology Leipzig, 1992-98 managment positions at building enterprises; Prof for construction and project management (1998). Research interests: Project management, facilities management, project development, information and communication processes, building contracts. 